\title{
Triplet excitation of $\mathrm{C}_{60}$ and the structure of the crystal at $1.2 \mathrm{~K}$
}

\author{
E.J.J. Groenen, O.G. Poluektov, M. Matsushita, J. Schmidt, J.H. van der Waals \\ Centre for the Study of Excited States of Molecules, Huygens Laboratory, University of Leiden. \\ P.O. Box 9504, 2300 RA Leiden, The Netherlands
}

and

\author{
G. Meijer \\ Department of Molecular and Laser Physics, University of Nijmegen, Toernooiveld, 6525 ED Nijmegen, The Netherlands
}

Received 2 June 1992

\begin{abstract}
Electron-spin-echo experiments at $95 \mathrm{GHz}$ and $1.2 \mathrm{~K}$ reveal that the triplet excitation of a single crystal of $\mathrm{C}_{60}$ is delocalized over either a pair or a chain of $\mathrm{C}_{60}$ molecules along $\langle 110\rangle$ directions. Two distinct triplet species have been observed in a one-toone ratio which verifies the proposed $2 a_{0}$ face-centred cubic superstructure of the crystal at low temperature. The $\mathrm{C}_{60}$ molecules on the two sublattices differ by a rotation over $60^{\circ}$ about $\langle 111\rangle$ directions.
\end{abstract}

\section{Introduction}

Quantum-chemical calculations on the icosahedral $\mathrm{C}_{60}$ molecule, buckminsterfullerene, suggest a $T_{1 \mathrm{~g}}$ or $T_{2 \mathrm{~g}}$ lowest triplet state [1]. The degeneracy of these electronically excited states makes them subject to Jahn-Teller instability. The results of Wasielewski et al. [2], who studied the triplet state of $\mathrm{C}_{60}$ in degassed toluene, support this expectation. They observed intense spin-polarized triplet EPR spectra and reported a zero-field splitting of the sublevels characterized by the parameters $|D|=0.0114$ $\mathrm{cm}^{-1}$ and $|E|=0.00069 \mathrm{~cm}^{-1}$ in the spin Hamiltonian which is incompatible with icosahedral symmetry. The nature of the distortion as well as its independence on the environment of the $\mathrm{C}_{60}$ molecule poses an intriguing question and led us to the study of the triplet excitation in a single crystal.

Structural studies of crystalline $\mathrm{C}_{60}$ have revealed phase transitions upon temperature lowering. At room temperature orientationally disordered $\mathrm{C}_{60}$

Correspondence to: E.J.J. Groenen, Centre for the Study of Excited States of Molecules, Huygens Laboratory, University of Leiden, P.O. Box 9504, 2300 RA Leiden, The Netherlands. molecules build up a face-centred cubic lattice [3]. Below $250 \mathrm{~K}$ the molecules become orientationally ordered and the structure has been reported to be simple cubic [4]. High-resolution powder neutron diffraction data indicate that for temperatures below $90 \mathrm{~K}$ rotational motion gets frozen but that some static disorder persists down to $5 \mathrm{~K}[5,6]$. Recent electron-diffraction studies [7] from $100 \mathrm{~K}$ down to $4 \mathrm{~K}$ point to a $2 a_{0}$ face-centred cubic superstructure, but the relative orientation of the $\mathrm{C}_{60}$ molecules in the sublattices could only be speculated upon.

Here we present the results of an electron-spin-echo (ESE) study at $95 \mathrm{GHz}$ on the triplet state of $\mathrm{C}_{60}$. A single crystal of submillimeter dimensions could be investigated owing to the sensitivity of the highfrequency ESE technique in combination with pulsed laser excitation. The data allow us to discuss both the triplet excitation and the structure of the crystal at $1.2 \mathrm{~K}$. The triplet excitation is found to be delocalized over either a pair or a chain of $\mathrm{C}_{60}$ molecules along $\langle 110\rangle$ directions. Two distinct triplets have been observed in accordance with the proposed superstructure and the orientation of the fine-structure principal axes of the two triplets allows for a determination of the orientation of the triplet molecules 
in the two sublattices of the superstructure.

\section{Experiments and results}

Single crystals of pure $\mathrm{C}_{60}$ were grown as described previously [ 8 ]. A crystal $\left(\approx 0.4 \times 0.4 \times 0.2 \mathrm{~mm}^{3}\right)$ was mounted in the cylindrical cavity of a $95 \mathrm{GHz}$ ESE spectrometer [9]. The crystal was cooled from room temperature to $200 \mathrm{~K}$ at a rate of $5 \mathrm{~K} / \mathrm{min}$, thereafter at a rate of $15 \mathrm{~K} / \mathrm{min}$. All experiments were performed at $1.2 \mathrm{~K}$. Samples were irradiated at 532 $\mathrm{nm}$ by the second harmonic of a Quanta Ray DCR$2 \mathrm{Nd}$ : YAG laser with $1 \mathrm{~mJ}$ pulses at a repetition rate of $8 \mathrm{~Hz}$. Two-pulse echo experiments were performed using $\pi / 2, \pi$ microwave excitation pulses of $50,100 \mathrm{~ns}$.

Strong ESE signals were observed and EPR spectra were recorded by monitoring the echo intensity while sweeping the magnetic field. The variation of such ESE-detected EPR spectra with the orientation of the magnetic field $B_{0}$ relative to the crystal revealed the presence of two distinct triplet species, $\alpha$ and $\beta$, with different fine-structure parameters but coinciding fine-structure principal axes systems $(x$, $y, z$ ). Each triplet species occurs in six magnetically distinguishable but otherwise equivalent sites (to be labeled by the subscripts 1 to 6 ), interrelated by the cubic symmetry of the crystal. Fig. 1 shows ESE-detected EPR spectra with $\boldsymbol{B}_{0}$ along stationary directions. The directions of the fine-structure principal axes relative to the crystal are illustrated in fig. 2 for sites 1 and 2. All $z$ axes lie along fourfold cube axes and if, as in fig. 2, $z$ is parallel to [001] then $x$ and $y$ lie parallel to [110] and [ $\overline{1} 10]$, or vice versa. Thus, for the spectrum in fig. 1a, with $B_{0}$ along [110], the magnetic field is simultaneously parallel to $x_{1}^{\alpha}$, (i.e. the direction of the principal $x$ axis of triplet $\alpha$ in site $1), y_{2}^{\mathbf{a}}, x_{1}^{\beta}$ and $y_{2}^{\beta}$. For the spectrum in fig. $1 \mathrm{~b}$ with $B_{0}$ along [001] the magnetic field is simultaneously parallel to $z_{1}^{\alpha}, z_{2}^{\alpha}, z_{1}^{\beta}$ and $z_{2}^{\beta}$. The signals at the stationary resonance fields have been labelled accordingly. The stationary resonance fields for $B_{0}$ parallel to $y$ coincide for triplets $\alpha$ and $\beta$; upon rotating the magnetic field away from [110] the corresponding ESE signal splits into two peaks of equal intensity. From the stationary resonance fields the principal values $X, Y, Z$ of the fine-structure tensors given in table 1 were obtained.

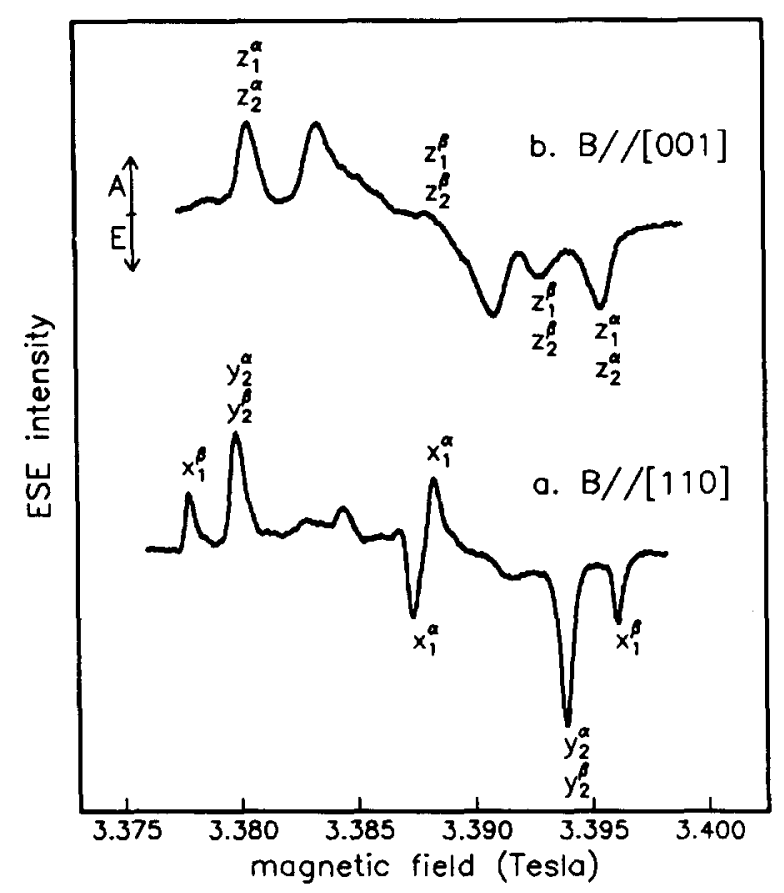

Fig. 1. ESE-detected EPR spectra for $\boldsymbol{B}_{0}$ (a) parallel to [110] and (b) parallel to [001]. $A$ refers to absorption, $E$ to emission of microwaves. The time between the laser flash and the first microwave pulse was $10 \mu \mathrm{s}$, the time between the exciting microwave pulses $1 \mu \mathrm{s}$. Labelled low-and high-field signals derive from sites for which the magnetic field is parallel to the indicated principal direction of the fine-structure tensor, while unlabelled signals derive from other sites.

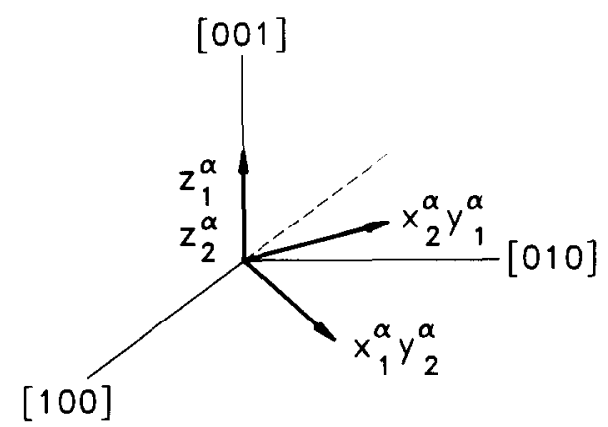

Fig. 2. Direction of the principal axes of the fine-structure tensor for sites 1 and 2 of triplet $\alpha$ with respect to the crystal axes. An identical picture holds for triplet $\beta$.

To obtain more insight into the triplet state of individual molecules, additional experiments were performed at $95 \mathrm{GHz}$ on $\mathrm{C}_{60}$ dissolved in decaline/ cyclohexane $(3 / 1)$ and in toluene $/ 10 \%$ poly $(\alpha-$ 
Table 1

Fine-structure parameters $(\mathrm{MHz})$ of $\mathrm{C}_{60}$ triplets in the single crystal and in toluene and decaline/cyclohexane glasses. The values in the last column have been calculated from $D$ and $E$ parameters given in ref. [2]. The angle $\alpha$ represents the angle between the $x^{A}$ and the $x^{\alpha}$ axis, the angle $\beta$ that between the $x^{A}$ and the $x^{\beta}$ axis. Both angles have been calculated from $X^{\varphi}=\cos ^{2} \varphi X^{8}+$ $\sin ^{2} \varphi Z^{8}$, where $\varphi=\alpha, \beta$ and $X^{g}$ and $Z^{g}$ represent the values of the parameters in the glasses. The indicated uncertainty in $\alpha$ and $\beta$ is an estimate based on a $5 \%$ variation in the values of $X^{\mathrm{B}}$ and $Z^{\mathrm{g}}$

\begin{tabular}{lccc}
\hline & \multicolumn{2}{l}{$\mathrm{C}_{60}$ single crystal } & $\mathrm{C}_{60}$ in glasses \\
\cline { 2 - 3 } & triplet $\alpha$ & $\operatorname{triplet} \beta$ & \\
\hline$|X| / h$ & $8 \pm 1$ & $171 \pm 1$ & 93 \\
$|Y| / h$ & $132 \pm 1$ & $132 \pm 1$ & 135 \\
$|Z| / h$ & $140 \pm 1$ & $39 \pm 1$ & 228 \\
& $\alpha=31^{\circ} \pm 2^{\circ}$ & $\beta=65^{\circ} \pm 2^{\circ}$ & \\
\hline
\end{tabular}

methylstyrene) at a concentration of $\approx 5 \times 10^{-4} \mathrm{M}$. The ESE-detected EPR spectra recorded in these two glasses were identical within experimental accuracy and consistent with the spectrum reported previously for $\mathrm{C}_{60}$ in degassed toluene at $9.3 \mathrm{GHz}$ [2]. The corresponding values for $X, Y, Z$, given in table 1 , have been inferred from ref. [2] because for the glasses their determination from the $95 \mathrm{GHz}$ spectra would be less accurate due to the effects of $g$-strain.

\section{Discussion}

Let us first consider the experiments in the dilute glasses. The identity of the triplet EPR spectra in the two glasses, in which the environment of the $C_{60}$ molecules differs considerably, points to a fine-structure that reflects an intrinsic property of the $\mathrm{C}_{60}$ molecule. Apparently, the stabilization energy that results from the Jahn-Teller deformation outweighs the solvation energy. Because the triplet state belongs to the irreducible representation $T_{1 g}$ or $T_{2 g}$ of $I_{h}$, the Jahn-Teller active coordinate is of $h_{g}$ symmetry [10]. This is compatible with a symmetry lowering of $\mathrm{C}_{60}$ on excitation from $I_{h}$ to $D_{2 h}$, the largest subgroup of $I_{h}$ that is in accord with the observed inequality of the zero-field energics $X, Y$ and $Z$. If the distortion of an individual molecule indeed results in $D_{2 h}$ symmetry - which we shall see to be in line with our crystal EPR results - the direction of the principal axes of the fine-structure tensor in the molecule is fixed by symmetry along three perpendicular twofold axes. The twofold axes of $\mathrm{C}_{60}$, of which there are fifteen, connect the midpoints of two opposite inter-pentagon bonds.

Now consider the crystal. It appears that the triplet states observed in the crystal do not concern traps of individual molecules. In that case we would have observed four (or a multiple of four) differentially oriented triplet species whose zero-field splitting reflected to some extent the $S_{6}$ symmetry of the crystal field [5]. Instead, the triplet states concern dimers or Frenkel excitons in which the excitation is delocalized over either a pair or a chain of $\mathrm{C}_{60}$ molecules. In order to bear out this conclusion we have to consider the crystal structure first.

Upon cooling, the $\mathrm{C}_{60}$ crystal undergoes a phase transition from face-centred cubic to simple cubic at $250 \mathrm{~K}$ where the quasi-freely rotating molecules become orientationally ordered $[4,5]$. The simple-cubic structure, space group $\mathrm{Pa} \overline{3}$, has been described as four interpenetrating simple-cubic sublattices, each sublattice being occupied by one of four types of differently oriented molecules A, B, C and D (fig. 3a). The label $\mathrm{A}$ refers to a $\mathrm{C}_{60}$ molecule that has an improper $S_{6}$ rotation axis along [111] and is rotated anticlockwise about this direction by an angle $\phi=98^{\circ}$ as compared to what its orientation would have been if the space group were $\mathrm{Fm} \overline{3}$ [3]. The other $\mathrm{C}_{60} \mathrm{~mol}-$ ecules, labelled $B, C$ and $D$, are similarly rotated through the same angle but about the other three $\langle 111\rangle$ directions. In this way all twelve nearestneighbour interactions along $\langle 110\rangle$ are simultaneously optimized, each contact corresponding to an electron-rich inter-pentagon bond facing an electronpoor pentagon centre. Later, it was suggested on the basis of refinements of powder neutron-diffraction data that a fraction of the $\mathrm{C}_{60}$ molecules assumes a different orientation with $\phi=38^{\circ}[6,11]$. The latter orientation corresponds to contacts of electron-rich inter-pentagon bonds facing electron-poor hexagon centres. Instead, Van Tendeloo et al. [7] recently observed a $2 a_{0}$ face-centred superstructure as shown in fig. $3 \mathrm{~b}$ and they speculated that the unprimed and primed sublattices might differ as regards the orientation of the $C_{60}$ molecules relative to their respective $\langle 111\rangle$ directions.

At this stage, our data do not allow us to draw con- 


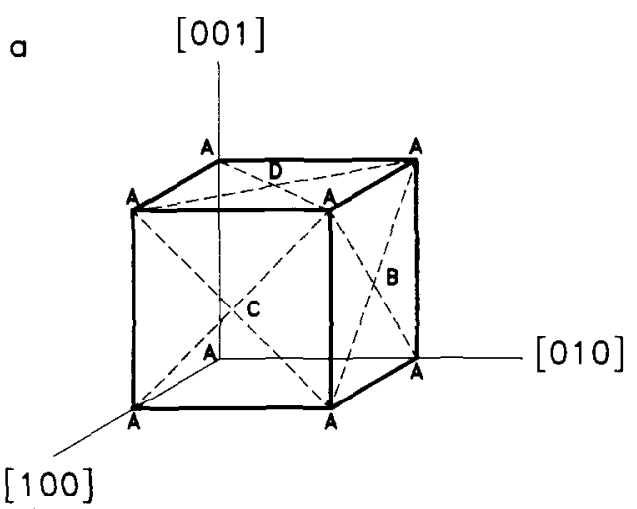

b

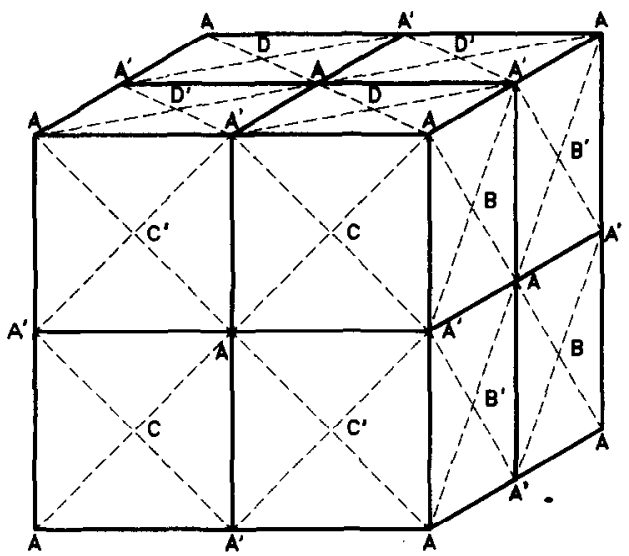

Fig. 3. Schematic representations of (a) the simple-cubic structure according to ref. [4] and (b) the face-centred cubic structure according to ref. [7]. The letters $A, B, C$ and $D$ refer to $\mathrm{C}_{60}$ molecules having a particular orientation relative to one of the <11 directions. Unprimed and primed letters correspond to different rotations about these directions (see text).

clusions as to the extent of the delocalization of the triplet excitation. Although the delocalization may well be confined to a pair of $\mathrm{C}_{60}$ molecules, we use from now on the terms "chain" and "exciton" for the sake of brevity. In order to assign triplet $\alpha$, consider the $A B$ chain along [101] in fig. 3a and suppose that the exchange interaction between $A$ and $B$ is strong enough for the triplet excitation to become delocalized. The direction of the fine-structure principal axes and the zero-field parameters of the exciton then should become averages of those of molecules $A$ and $B$. Our observation that the value of $Y$ in the crystal is virtually equal to that in the glass suggests that the molecules $A$ and $B$ have their $y$ axes parallel. Inspection of the crystal structure shows that
$A$ and $B$ in their ground-state orientation [4,5] both have a $C_{2}$ axis along [110], which corresponds to the direction of the $y$ axis found for one of the type $\alpha$ triplets, $y_{1}^{\alpha}$ in fig. 2. The principal $y$ axis for the $A B$ exciton thus makes an angle of $60^{\circ}$ with the chain direction. The corresponding principal $x$ and $z$ axes of molecules A and B lie in the ( $\overline{1} 10)$ plane. This is schematically indicated in fig. 4 where we have drawn for the molecules A and B in their ground-state orientation the cross sections in the mirror planes perpendicular to their common $y$ axes. Averaging of the zero-field tensors of the A and B molecules leads to $x_{1}^{\alpha}$ and $z_{1}^{\alpha}$ axes of the exciton along [110] and [001] in agreement with our observation. Assuming that the observed values $X^{\alpha}$ and $Z^{\alpha}$ for the exciton result from averaging of the corresponding $X$ and $Z$ parameters for the individual $\mathrm{C}_{60}$ molecules in a glass and using our former label $\alpha$ to denote the angle between the $x^{\mathrm{A}}\left(x^{\mathrm{B}}\right)$ and the $x^{\alpha}$ axis, we calculate $\alpha=31^{\circ} \pm 2^{\circ}$ (table 1 ). This value for $\alpha$ nicely corroborates our model because in the ground-state orientation the $C_{2}$ axis makes an angle of about $33.5^{\circ}$ with [110] (cf. fig. 4). In summary, both the directions of the principal axes of the fine-structure tensor and the values of the fine-structure parameters ob-

a)

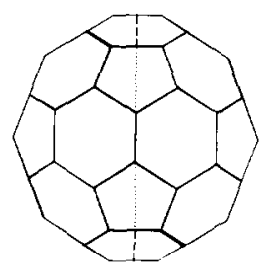

b)

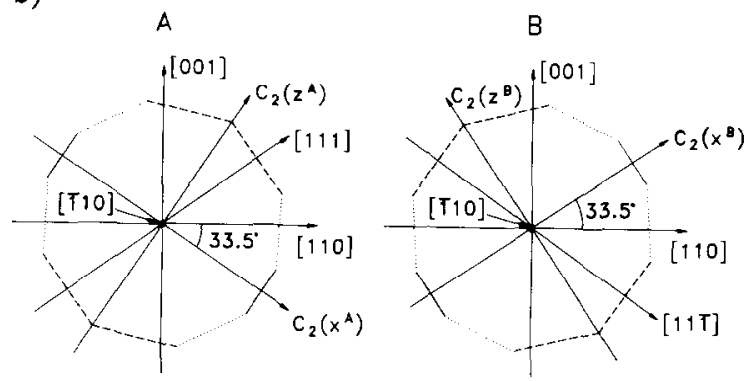

Fig. 4. (a) Buckminsterfullerene $C_{60}$. (b) Cross sections for $C_{60}$ molecules $A$ and $B$ in the reflection planes perpendicular to $[\overline{1} 10]$. The orientations are as in the ground state according to the simple cubic structure. The drawn edges of the decagon are defined in fig. $4 a$. 
served for triplet $\alpha$ in site 1 support the interpretation of the triplet excitation as being delocalized along the AB chain in the crystal. Similar considerations apply to the other sites of triplets $\alpha$ which correspond to the remaining five combinations of two molecular orientations from the set $\mathrm{A}, \mathrm{B}, \mathrm{C}, \mathrm{D}$.

Because all chains along the $\langle 110\rangle$ directions in the simple-cubic lattice (fig. $3 \mathrm{a}$ ) are equivalent, such a structure does not allow for a second distinct triplet. In the $2 a_{0}$ face-centred cubic superstructure (fig. $3 b)$ triplet $\beta$ occurs naturally as an exciton delocalized over a chain in the primed sublattice, e.g. $A^{\prime} B^{\prime}$. From the orientation of the principal axes and the values of the fine-structure parameters for triplet $\beta$ we conclude that the primed $\mathrm{C}_{60}$ molecules differ from the unprimed ones by a rotation over $60^{\circ}$ about their $\langle 111\rangle$ directions. This is the only orientation, besides that of the unprimed molecules, for which again two $C_{2}$ axes, now of $\mathrm{A}^{\prime}$ and of $\mathrm{B}^{\prime}$, coincide along [ 110$]$. This makes the value of $Y^{\beta}$ equal to that of $Y$ in the glass, as for $Y^{\alpha}$. The values of $X^{\beta}$ and $Z^{\beta}$ become different from those of $X^{\alpha}$ and $Z^{\alpha}$ because the $C_{2}$ axes of the $\mathrm{A}^{\prime}$ and $\mathrm{B}^{\prime}$ molecules in the (110) plane are oriented differently with respect to [110] and [001] as compared to those of A and B.

The relative orientation of neighbouring $\mathrm{C}_{60}$ molecules which results from the above interpretation implies that the close contacts along the chains of primed molecules concern inter-pentagon bonds facing hexagon centres, while those along the chains of unprimed molecules concern inter-pentagon bonds facing pentagon centres. The orientation found for the primed $\mathrm{C}_{60}$ molecules in the superstructure corresponds to that postulated by David et al. [6] and $\mathrm{Hu}$ et al. [11] for the fraction of disordered molecules to which they concluded on the basis of powder neutron diffraction data. However, our observations really point to a $2 a_{0}$ superstructure because the ESE signals for $\boldsymbol{B}_{0}$ parallel $\boldsymbol{y}$ (cf. fig. 1a) result from signals of triplet $\alpha$, delocalized along an unprimed chain, and of triplet $\beta$, delocalized along a primed chain, in a one-to-one ratio.

Thus, under the plausible assumption that the triplet excitation does not appreciably change the structure, our electron-spin-echo experiments confirm that the crystal structure at $1.2 \mathrm{~K}$ is cubic with a $2 a_{0}$ face-centered superstructure. In addition they have revealed that the unprimed and primed sub- lattices differ by a rotation over $60^{\circ}$ of the $\mathrm{C}_{60}$ molecules about $\langle 111\rangle$ directions. The triplet excitation of the crystal is found to be delocalized, but the present experiment does not discriminate between a delocalization over either a pair or a chain of $\mathrm{C}_{60} \mathrm{~mol}$ ccules. The idea that $\mathrm{C}_{60}$ may be a model system for one-dimensional triplet excitons is fascinating and stimulates further research.

\section{Acknowledgement}

We gratefully acknowledge M.A. Verheijen for his assistance in the preparation of the $\mathrm{C}_{60}$ crystals and Dr. H.T. Jonkman for a gift of pure $\mathrm{C}_{60}$ powder. This work was supported by The Netherlands Foundation for Fundamental Research on Matter (FOM) and by The Netherlands Foundation for Chemical Research (SON) with financial aid from The Netherlands Organization for Scientific Research (NWO).

\section{References}

[1] F. Negri, G. Orlandi and F. Zerbetto, Chem. Phys. Letters 144 (1988) 31.

[2] M.R. Wasielewski, M.P. O'Neil, K.R. Lykke, M.J. Pellin and D.M. Gruen, J. Am. Chem. Soc. 113 (1991) 2774.

[3] R.M. Fleming, T. Siegrist, P.M. Marsh, B. Hessen, A.R. Kortan, D.W. Murphy, R.C. Haddon, R. Tycko, G. Dabbagh, A.M. Mujsce, M.L. Kaplan and S.M. Zahurak, Mater. Res. Soc. Symp. Proc. 206 (1991) 691.

[4] P.A. Heiney, J.E. Fischer, A.R. McGhie, W.J. Romanow, A.M. Denenstein, J.P. McCauley Jr., A.B. Smith III and D.E. Cox, Phys. Rev. Letters 66 (1991) 2911;

R. Sachidanandam and A.B. Harris, Phys. Rev. Letters 67 (1991) 1467.

[5] W.I.F.' David, R.M. Ibberson, J.C. Matthewman, K. Prassides, T.J.S. Dennis, J.P. Hare, H.W. Kroto, R. Taylor and D.R.M. Walton, Nature 353 (1991) 147.

[6] W.I.F. David, R.M. Ibberson, T.J.S. Dennis, J.P. Hare and K. Prassides, Europhys. Letters 18 (1992) 219.

[7] G. van Tendeloo, S. Amelinckx, M.A. Verheijen, P.H.M. van Loosdrecht and G. Meijer, Phys. Rev. Letters, submitted for publication.

[8] M.A. Verheijen, H. Meekes, G. Meijer, E. Raas and P. Bennema, Chem. Phys. Letters 191 (1992) 339.

[9] R.T. Weber, J.A.J.M. Disselhorst, L.J. Prevo, J. Schmidt and W.Th. Wenckebach, J. Magn. Reson. 81 (1989) 129.

[10] G. Herzberg, Electronic spectra and electronic structure of polyatomic molecules (Van Nostrand, Princeton, 1966) p. 50.

[11] R. Hu, T. Egami, F. Li and J.S. Lannin, Phys. Rev. B 45 (1992) 9517. 